\title{
Factors controlling the vesicular-arbuscular mycorrhizal (VAM) infection of roots
}

\author{
CHRISTINE HEPPER
}

The extent of VAM infection of a root system depends on the host, fungus and environment, in particular the soil, which will affect the host nutrient status, the regeneration of fungal propagules and their success in colonizing the roots.

In an experiment to study the interaction between phosphate and nitrate on VAM infection, it was found that increasing levels of phosphate decreased infection, whereas higher levels of nitrate resulted in denser infections and a greater proportion of the root system colonized. The amount of infection was influenced by the N:P ratio in the plant nutrient solution and the nitrogen and phosphorus content of the roots achieved by the different treatments. An N:P ratio of about 50 was required to give optimum infection levels in Lactuca sativa and Allium porrum. The effect of phosphate on root infection is thought to be mediated via the host plant's physiology and this is supported by the observation that phosphate itself does not inhibit the germination and growth of VAM fungal spores to any extent on agar or in soil.

By transplanting seedlings from a sterilised soil to one containing VAM inoculum, it has been shown that roots of Trifolium parviflorum lose their susceptibility to infection when less than 5 days old whereas those of Allium porrum can be infected at least up to 32 days.

The extent of colonization of Allium porrum by pairs of fungi, one placed to simulate an indigenous fungus, the other an introduced inoculum, has been investigated using the mobility of diagnostic enzymes during electrophoresis to identify the species colonizing the roots. The three species of Glomus used were very competitive in that one could totally exclude another from the root system. The success of a fungus in infecting a root system depended on its position in the competitive hierarchy and its placement in the soil.

All of the factors discussed must be considered if crop yields are to be improved by inoculation with VAM fungi.

Christine M. Hepper, Institute of Arable Crops Research, (Rothamsted) Harpenden, Hertfordshire, AL5 2JQ, UK 